\title{
LOW-COMPLEXITY TIME-DOMAIN ICI EQUALIZATION FOR OFDM COMMUNICATIONS OVER RAPIDLY VARYING CHANNELS
}

\author{
Tomasz Hrycak* and Gerald Matz ${ }^{\dagger}$ \\ *Numerical Harmonic Analysis Group (NuHAG), Dept. of Mathematics, University of Vienna \\ Nordbergstrasse 15, A-1090 Wien (Austria), Tel.: +43 14277 50655, Email: tomasz.hrycak@univie.ac.at \\ ${ }^{\dagger}$ Institute of Communications and Radio-Frequency Engineering, Vienna University of Technology \\ Gusshausstrasse 25/389, A-1040 Vienna (Austria), Tel.: +43 158801 38916, Email: gmatz@nt.tuwien.ac.at
}

\begin{abstract}
We consider wireless OFDM communications over rapidly varying channels as occurring e.g. in high-mobility scenarios. Here, intercarrier interference (ICI) is the major source of impairment that limits the transmission quality. In this paper, we propose novel equalizers to combat ICI and enable reliable communications even at high Doppler frequencies. In contrast to existing frequency-domain approaches, our schemes operate in the time domain. This allows a low-complexity implementation without introducing any systematic channel approximation errors. Numerical simulations illustrate the effectiveness of our approach and show that in conjunction with channel coding our methods achieve significant Doppler diversity.
\end{abstract}

\section{INTRODUCTION}

\subsection{Background and Motivation}

Orthogonal frequency division multiplexing (OFDM) is a communication scheme gaining more and more importance in wireless systems [1]. Examples include the WLAN and WMAN standards IEEE 802.11 and 802.16, respectively, as well as broadcasting applications like DVB-T, DAB, and DRM [2-6]. Usually, OFDM systems are designed so that no channel variations occur within the individual OFDM symbols. Recently, however, there has been increasing interest in rapidly varying channels, i.e., scenarios where the channel changes noticeably within an OFDM symbol (equivalently, channel coherence time is less than the OFDM symbol duration). In such situations, the underlying large Doppler shifts cause strong intercarrier interference (ICI), which becomes a major source of transmission impairment (apart from fading and noise). The reasons for the occurrence of rapidly varying channels are increased user mobility or non-negligible carrier frequency offsets. An example of recent practical relevance is the mobile reception of DVB-T (e.g. [7]), which was originally conceived for fixed receivers.

This work was supported by the WWTF project MOHAWI (MA 44) and the EU project MEXT-CT-2004-517154.

\subsection{Previous Work}

For severe ICI, conventional single-tap equalization in the frequency (subcarrier) domain breaks down in the sense that bit or symbol error rates saturate at high SNRs. The reason for this is the fact that above a certain SNR threshold, the ICI dominates the noise, i.e., the transmission is interference limited (see [8-11] for an analysis of the impact of ICI on receiver performance).

Motivated by this fact, several approaches have been proposed to combat ICI in OFDM transmissions over rapidly varying channels. In this paper, we focus on solutions that involve receiver processing only in order to remain compliant with existing OFDM systems. We note that all of the corresponding ICI mitigation techniques proposed so far operate in the frequency domain. In [12], minimum mean-square error (MMSE) and successive interference cancellation equalizers have been developed that use all subcarriers simultaneously. In [11], MMSE and decision-feedback equalizers involving only a few neighboring subcarriers have been presented in order to reduce computational complexity. The idea of using only a few subcarriers for equalization amounts to approximating the frequency-domain channel matrix by a banded matrix and has been further exploited for equalizer design using the LDL factorization in [13]. An iterative MMSE equalizer preceded by an "ICI-shaping" stage that concentrates the ICI power within a small band of the channel matrix is described in [14]. Alternative frequency-domain equalization approaches that model the channel variations using a firstorder polynomial are described in $[7,15]$.

\subsection{Contributions}

The paper contains the following original contributions:

- We propose to perform linear equalization and ICI mitigation in the time domain instead of the frequency domain, using the actual receive signal before FFT demodulation. This has the advantage that the channel matrix in the time domain is strictly banded.

- We develop low-complexity equalizer implementations using two regularization algorithms (cf. [16]). Both 
schemes exploit the band structure of the channel matrix and have complexity scaling linearly with the number of subcarriers.

- The first approach is time-domain MMSE equalization, which we show to be mathematically equivalent to frequency-domain MMSE equalization but which features significantly lower complexity.

- The second proposed equalizer is based on the LSQR algorithm [17], which is an efficient and numerically stable implementation of the iterative conjugate gradient method for normal equations (CGNE). Here, regularization is achieved by early termination of the iterations.

- We show bit error rate (BER) simulations for a convolutionally encoded OFDM system incorporating our equalization techniques. These simulations confirm the effectiveness of our schemes and illustrate their superiority over conventional single-tap equalization. Furthermore, in conjunction with the channel code our methods are seen to achieve significant Doppler diversity.

\section{SYSTEM MODEL}

We consider transmission over a time-varying frequency-selective fading channel characterized by its impulse response $h[n, m]$. We assume that the channel has maximum delay $L-1$, i.e., $h[n, m]=0$ for $m \neq 0, \ldots, L-1$. We do not make any assumptions regarding the amount of time-variation (equivalently, Doppler frequency) in the channel. For a classical OFDM system with cyclic prefix (CP) duration $L_{\mathrm{cp}} \leq L$, all OFDM symbols can be processed separately. Hence, in the subsequent discussion we only consider the first OFDM symbol for simplicity.

We consider a CP-OFDM system with $K$ subcarriers which results in a symbol duration of $N=K+L_{\mathrm{cp}}$. The length- $K$ vector $\mathbf{a}=\left(a_{0}, \ldots, a_{K-1}\right)^{T}$ containing the frequency-domain OFDM transmit symbols $a_{k}$ is obtained from the information bits via convolutional encoding, interleaving, and symbol mapping using a finite symbol alphabet $\mathcal{A}$. The OFDM modulator constructs the time-domain transmit signal using an inverse discrete Fourier transform as

$$
x[n]=\frac{1}{\sqrt{N}} \sum_{k=0}^{K-1} a_{k} e^{j 2 \pi \frac{n k}{K}}, \quad n=-L_{\mathrm{cp}}, \ldots, K-1 .
$$

We define the length- $K$ vector $\mathbf{x}=(x[0], \ldots, x[K-1])^{T}$ and the $K \times K$ DFT matrix $\mathbf{F}=\frac{1}{\sqrt{N}}\left[e^{-j 2 \pi \frac{k n}{K}}\right]_{n, k=0, \ldots, K-1}$, and rewrite (1) compactly as

$$
\mathbf{x}=\mathbf{F}^{H} \mathbf{a}
$$

(here, superscript ${ }^{H}$ denotes Hermitian transposition).
When sending $x[n]$ over the channel, the receive signal equals

$$
y[n]=\sum_{m=0}^{L-1} h[n, m] x[n-m]+w[n] .
$$

with $w[n]$ denoting stationary white Gaussian noise of variance $\sigma^{2}$. Discarding the CP (i.e., retaining only $y[n], n=$ $0, \ldots, K-1)$ and using the fact that $x[n]=x[n+K]$, $n=-L_{\mathrm{cp}}, \ldots, 1,(3)$ can be rewritten as

$$
\mathbf{y}=\mathbf{H x}+\mathbf{w} .
$$

Here, $\mathbf{H}$ is the $K \times K$ time-domain channel matrix with elements $[\mathbf{H}]_{k l}=h\left[k-1,(k-l)_{K}\right], k, l=0, \ldots, K-1$, where $(\cdot)_{K}$ denotes modulo- $K$ operation; furthermore, $\mathbf{y}=$ $(y[0], \ldots, y[K-1])^{T}$ and $\mathbf{w}=(w[0], \ldots, w[K-1])^{T}$. A fundamental observation at this point is that the channel matrix $\mathbf{H}$ has only $K L$ nonzero elements. Since $L \leq L_{\mathrm{cp}} \ll K$, this means that $\mathbf{H}$ is a sparse matrix.

The OFDM demodulator calculates the receive sequence according to $\tilde{y}_{k}=\frac{1}{\sqrt{N}} \sum_{n=0}^{K-1} y[n] e^{-j 2 \pi \frac{n k}{K}}$. Defining $\tilde{\mathbf{y}}=$ $\left(\tilde{y}_{0}, \ldots, \tilde{y}_{K-1}\right)^{T}$ and using (2), (4), we obtain the frequencydomain input-output relation

$$
\tilde{\mathbf{y}}=\mathbf{F y}=\tilde{\mathbf{H}} \mathbf{a}+\tilde{\mathbf{w}} .
$$

Here, the frequency-domain channel matrix is given by $\tilde{\mathbf{H}}=$ $\mathbf{F H F}{ }^{H}$ and $\tilde{\mathbf{w}}=\mathbf{F} \mathbf{w} \sim \mathcal{N}\left(\mathbf{0}, \sigma^{2} \mathbf{I}\right)$.

In the case of a time-invariant channel, $\mathbf{H}$ is a cyclic matrix and hence $\tilde{\mathbf{H}}$ is diagonal, i.e., there is no ICI. In this case, single-tap equalization is sufficient. In the time-varying case $\tilde{\mathbf{H}}$ is no longer diagonal and significant ICI can occur. For low to medium Doppler, however, $\tilde{\mathbf{H}}$ has noticeable off-diagonal decay and can hence be reasonably approximated by a banded matrix [11]. The number of off-diagonals to be taken into account depends on the maximum Doppler frequency and the desired approximation accuracy.

\section{PROPOSED EQUALIZERS}

Current state-of-the art to combat ICI uses frequency-domain equalization with equalizer designs using a band matrix approximation of $\tilde{\mathbf{H}}[11,13,14]$. This is motivated by the scalar per-carrier equalizers used in the time-invariant case. This approach introduces a systematic error and is useful (in terms of complexity) only for low-to-medium Doppler frequency.

In this paper, we propose to perform equalization in the time domain. This amounts to solving (4) in the leastsquares or minimum mean-square error sense for $\mathbf{x}$. The time-domain channel matrix $\mathbf{H}$ is already a sparse, banded matrix, with only $K L$ nonzero elements. Hence, systematic errors caused by channel approximations can be avoided and efficient equalizer implementations can be obtained. The output signal $\hat{\mathbf{x}}$ of the time-domain equalizer is transformed to the subcarrier domain, $\hat{\mathbf{a}}=\mathbf{F} \hat{\mathbf{x}}$, quantized, demapped, deinterleaved, and finally decoded. 
Since the channel matrix $\mathbf{H}$ can have a very high condition number (particularly in the high delay and high Doppler regimes), solutions using ZF equalization perform poorly and regularization techniques (cf. [16]) have to be considered. Below we investigate two approaches: MMSE equalization and equalization based on the LSQR algorithm [17]. Both schemes are computational efficient and achieve reasonable trade-offs between ICI equalization and noise enhancement. We note that equalization schemes exploiting the finite alphabet property (e.g., Viterbi detection, decision feedback equalization) cannot be used since in our context the symbols live in another domain than the domain where we equalize.

\subsection{MMSE Equalizer}

Time-domain MMSE ICI equalization amounts fo finding the linear equalizer matrix $\mathbf{W}$ such that $\mathbf{W y}$ is closest to $\mathbf{x}$ in the MMSE sense, i.e.,

$$
\mathbf{W}_{\mathrm{MMSE}}=\arg \min _{\mathbf{W}} \mathcal{E}\left\{\|\mathbf{W} \mathbf{y}-\mathbf{x}\|^{2}\right\} .
$$

The solution of this minimization problem is straightforwardly obtained as

$$
\mathbf{W}_{\text {MMSE }}=\left(\mathbf{H}^{H} \mathbf{H}+\sigma^{2} \mathbf{I}\right)^{-1} \mathbf{H}^{H} .
$$

The MMSE equalizer output equals

$$
\hat{\mathbf{x}}_{\text {MMSE }}=\mathbf{W}_{\text {MMSE }} \mathbf{y}=\left(\mathbf{H}^{H} \mathbf{H}+\sigma^{2} \mathbf{I}\right)^{-1} \mathbf{H}^{H} \mathbf{y} .
$$

Since $\mathbf{H}$ is sparse with $K L$ nonzero elements, it can be shown that $\mathbf{H}^{H} \mathbf{H}$ is sparse as well with $K(2 L-1)$ nonzero elements, which can be computed using $2 K L^{2}$ flops. The overall complexity of computing $\hat{\mathbf{x}}$ can be shown to require $\mathcal{O}\left(K L^{2}\right)$ operations and hence scales only linearly with the number of subcarriers $K$.

The MMSE equalizer output $\hat{\mathbf{x}}_{\mathrm{MMSE}}$ is mapped to the frequency domain as $\mathbf{F} \hat{\mathbf{x}}_{\mathrm{MMSE}}$ and afterwards quantized with respect to the symbol alphabet. Since the DFT matrix $\mathbf{F}$ is unitary, $\mathbf{F} \hat{\mathbf{x}}_{\text {MMSE }}$ equals the solution of frequency-domain MMSE equalization. Indeed, using $\mathbf{I}=\mathbf{F}^{H} \mathbf{F}=\mathbf{F F}^{H}$ and $\mathbf{F}^{-1}=\mathbf{F}^{H}$ we obtain

$$
\begin{aligned}
\mathbf{F} \hat{\mathbf{x}}_{\mathrm{MMSE}} & =\mathbf{F}\left(\mathbf{H}^{H} \mathbf{H}+\sigma^{2} \mathbf{I}\right)^{-1} \mathbf{H}^{H} \mathbf{F}^{H} \mathbf{F} \mathbf{y} \\
& =\left(\mathbf{F}\left(\mathbf{H}^{H} \mathbf{F}^{H} \mathbf{F} \mathbf{H}+\sigma^{2} \mathbf{I}\right) \mathbf{F}^{H}\right)^{-1} \mathbf{F} \mathbf{H}^{H} \mathbf{F}^{H} \mathbf{F} \mathbf{y} \\
& =\left(\tilde{\mathbf{H}}{ }^{H} \tilde{\mathbf{H}}+\sigma^{2}\right)^{-1} \tilde{\mathbf{H}}^{H} \tilde{\mathbf{y}} .
\end{aligned}
$$

We conclude that time-domain and frequency-domain MMSE equalization are mathematically equivalent and result in the same performance. However, full-blown frequency-domain MMSE equalization in general has complexity $\mathcal{O}\left(K^{3}\right)$. Using a frequency domain band approximation, complexity equals $\mathcal{O}\left(K B^{2}\right)$, where $B$ denotes the number of diagonals used in the band approximation. Hence, in the high-Doppler regime where $B>L$, time-domain MMSE equalization should be preferred over frequency-domain MMSE equalization due to lower complexity and the fact that no systematic band approximation error is present.

\subsection{LSQR Equalizer}

LSQR is an iterative algorithm for solving least squares problem specifically tailored to sparse matrices [17]. It is equivalent to the conjugate gradient method for the normal equations (CGNE) $\mathbf{H}^{H} \mathbf{H x}=\mathbf{H}^{H} \mathbf{y}$ but has better numerical properties. The normal equations are obtained from (4) by leftmultiplying by $\mathbf{H}^{H}$ and omitting the noise. One can show that in the $i$ th LSQR iteration, an approximate solution of the normal equations is obtained by minimizing $\left\|\mathbf{H x}_{i}-\mathbf{y}\right\|^{2}$ subject to the constraint that $\mathbf{x}_{i}$ lies in the Krylov subspace ${ }^{1}$ $\mathcal{K}\left(\mathbf{H}^{H} \mathbf{H}, \mathbf{H}^{H} \mathbf{y}, i\right)$. The actual LSQR implementation involves Golub-Kahan bidiagonalization [18] and a cheap QR decomposition to solve the least-squares problem (see the Appendix for further details). A close look at the LSQR implementation reveals that each iteration requires $\mathcal{O}(K L)$ flops.

We note that although LSQR is described via Krylov subspaces generated by $\mathbf{H}^{H} \mathbf{H}$, its performance is actually governed by the condition number $\kappa(\mathbf{H})$ of $\mathbf{H}$ and not by the condition number of $\mathbf{H}^{H} \mathbf{H}$ which is the square of $\kappa(\mathbf{H})$. This property is crucial, since large condition numbers of $\mathbf{H}$ occur frequently in the high Doppler and high delay regimes. The latter is also the reason why regularization is inevitable. With LSQR, regularization is achieved via an early termination of the iteration process (see e.g. [16]). The regularizing behavior is due to the fact that the initial iterations reduce the approximation error $\mathbf{H x}_{i}-\mathbf{y}$ in the directions of the dominant right singular vectors of $\mathbf{H}$, which are less affected by noise than the directions corresponding to small singular values. There is an optimum number $I$ of LSQR iterations (with regard to the error $\left\|\mathbf{H x}_{i}-\mathbf{y}\right\|^{2}$ ) for which most of the channel distortions are equalized while noise enhancement simultaneously is low. Fewer iterations result in larger residual ICI and more iterations cause more pronounced noise enhancement. However, the corresponding minimum usually is not very pronounced, i.e., doing slightly more or slightly less than $I$ iterations does not change the result dramatically. We further observed that the optimum number of iterations tends to grow slowly with increasing Doppler but keeping the number of iterations fixed does not degrade performance noticeably.

We conclude that LSQR is particularly attractive due to its numerical stability, inherent potential for regularization, and low computational complexity of $\mathcal{O}(K L I)$ flops in total. For $I<L$, the LSQR equalizer is even more efficient than the MMSE equalizer discussed in Section 3.1. In Section 4 we will furthermore see that LSQR has the potential to significantly outperform MMSE equalization.

\section{SIMULATION RESULTS}

\subsection{Simulation Setup}

We present numerical simulations for an OFDM system with $K=256$ subcarriers and a CP length of $L_{\mathrm{cp}}=16$ samples.

\footnotetext{
${ }^{1}$ The Krylov subspace $\mathcal{K}(\mathbf{A}, \mathbf{b}, i)$ is defined as the span of $\left\{\mathbf{b}, \mathbf{A b}, \ldots, \mathbf{A}^{i} \mathbf{b}\right\}$; see [18, Section 9.1.1] for further details.
} 


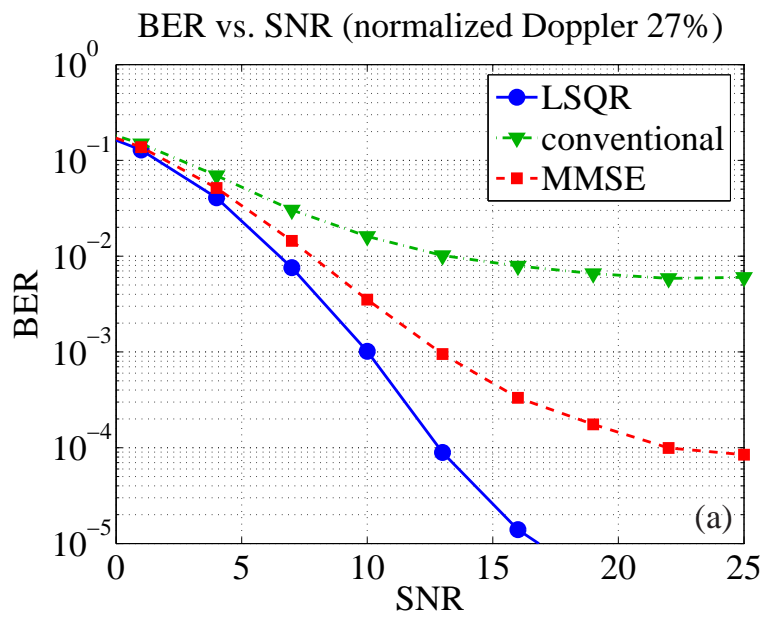

BER vs. normalized Doppler $(\mathrm{SNR}=17 \mathrm{~dB})$

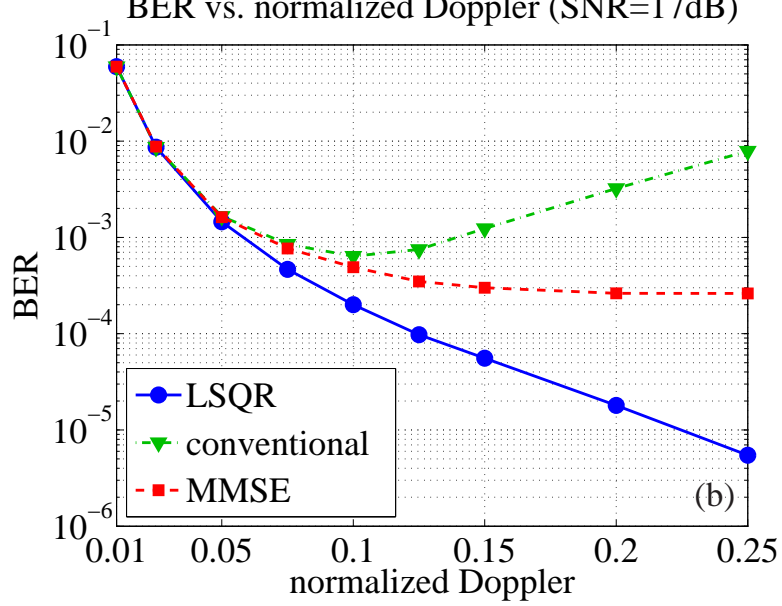

Fig. 1. Comparison of time-domain LSQR and MMSE equalization with conventional single-tap equalization: (a) BER versus SNR for $27 \%$ normalized Doppler, (b) BER vs. normalized Doppler for an SNR of $17 \mathrm{~dB}$.

We used a rate $1 / 2$ convolutional code (generator polynomial $\left.\left(13_{8}, 15_{8}\right)\right), 32 \times 16$ row-column interleaving, and a 4-QAM symbol alphabet with Gray labeling. A wide-sense stationary uncorrelated scattering channel [19] with a uniform delay and Doppler profile was simulated according to [20]. The maximum delay of the channel was $L=10$ and maximum normalized Doppler (physical Doppler frequency divided by subcarrier spacing) ranged from $1 \%$ to $27 \%$. For the LSQR equalizer, we used a fixed number of $I=15$ iterations.

\subsection{BER versus SNR}

For a normalized Doppler spread of $27 \%$, Fig. 1(a) compares the coded bit error rates (BERs) versus SNR obtained with our proposed equalizers and a conventional single-tap equalizer that ignores ICI. It is seen that single-tap equalization strongly suffers from residual ICI, which results in a BER saturation at $6 \cdot 10^{-3}$ at SNRs larger than $20 \mathrm{~dB}$. In contrast, our low-complexity methods succeed in combatting ICI and thus outperform single-tap equalization by orders of magnitude. At an SNR of $15 \mathrm{~dB}$, conventional equalization yields a BER of $9 \cdot 10^{-3}$, whereas our proposed MMSE and LSQR equalizers achieve a BER of $5 \cdot 10^{-4}$ and $3 \cdot 10^{-5}$, respectively.

Furthermore, for the case considered, LSQR equalization achieves better performance than MMSE equalization. To achieve $10^{-4}$ BER, MMSE equalization requires an SNR of $22 \mathrm{~dB}$ whereas LSQR achieves the same performance at an SNR of only $13 \mathrm{~dB}$. This performance advantage can be explained by the observation that LSQR initially tends to correct only the channel phases (apart of reducing ICI), which in turn allows reliable recovery of the 4-QAM symbols with very small noise enhancement. This advantage of LSQR will be less pronounced, however, for higher-order symbol constellations.

\subsection{BER versus Doppler}

Fig. 1(b) shows coded BER versus normalized Doppler frequency at a fixed SNR of $17 \mathrm{~dB}$. For normalized Doppler frequencies below $5 \%$ all methods have essentially identical performance. For increasing Doppler, however, single-tap equalization breaks down quickly and BER deteriorates significantly since ICI is not mitigated. On the other hand, the BER achieved with MMSE and LSQR equalization decreases with growing Doppler (only weakly, however, for the MMSE equalizer). This is due to the fact that in conjunction with the channel code our schemes allow to exploit large amounts of Doppler diversity in addition to delay diversity [21]. Again, the LSQR approach is seen to outperform MMSE equalization whose BER saturates at $2 \cdot 10^{-4}$ whereas the BER of LSQR decreases with almost constant slope. We note that this performance gain of LSQR comes at no extra computational costs since for fixed iteration number LSQR complexity does not depend on the Doppler frequency. For 25\% normalized Doppler, LSQR equalization (BER $6 \cdot 10^{-6}$ ) outperforms conventional equalization (BER $8 \cdot 10^{-3}$ ) by more than three orders of magnitude.

\section{CONCLUSIONS}

We proposed two time-domain ICI equalization schemes for OFDM systems operating over channels with rapid time-variations. Such scenarios occur e.g. with the mobile reception of DVB-T. Our time-domain approach has the advantage of allowing for low-complexity implementations since the channel corresponds to a sparse matrix. Moreover, systematic errors can be avoided because no channel approximation needs to be performed. In addition to MMSE equalization, we introduced an iterative conjugate gradient based equalizer using the LSQR algorithm. Apart from low complexity that scales linearly with the number of subcarriers, our equalizers show excellent performance and even allow to realize significant Doppler diversity in conjunction with channel coding. 


\section{APPENDIX: LSQR}

LSQR is an iterative algorithm for approximate solution of the linear system $\mathbf{H x}=\mathbf{y}$ [17]. In exact arithmetic, it is equivalent to the conjugate gradient method for the normal equations $\mathbf{H}^{H} \mathbf{H x}=\mathbf{H}^{H} \mathbf{y}$ (CGNE). However, it has practical advantages regarding complexity and numerical stability. Specifically, in the $i$ th iteration, the LSQR algorithm constructs a vector $\mathbf{x}_{i}$ in the Krylov subspace $\mathcal{K}\left(\mathbf{H}^{H} \mathbf{H}, \mathbf{H}^{H} \mathbf{y}, i\right)$ that minimizes $\left\|\mathbf{H x}_{i}-\mathbf{y}\right\|^{2}$. The actual implementation consists of two steps: the Golub-Kahan bidiagonalization and solution of a bidiagonal least squares problem.

The Golub-Kahan bidiagonalization [18] constructs vectors $\mathbf{u}_{i}, \mathbf{v}_{i}$, and positive constants $\alpha_{i}, \beta_{i}$ as follows:

1. Initialization:

$$
\begin{array}{rlrl}
\beta_{1} & =\|\mathbf{y}\|, & & \alpha_{1}=\left\|\mathbf{H}^{H} \mathbf{y}\right\|, \\
\mathbf{u}_{1}=\mathbf{y} / \beta_{1}, & \mathbf{v}_{1}=\mathbf{H}^{H} \mathbf{y} / \alpha_{1} .
\end{array}
$$

2. Recursively compute

$$
\begin{gathered}
\beta_{i+1}=\left\|\mathbf{H} \mathbf{v}_{i}-\alpha_{i} \mathbf{u}_{i}\right\|, \\
\alpha_{i+1}=\left\|\mathbf{H}^{H} \mathbf{u}_{i}-\beta_{i} \mathbf{v}_{i}\right\|, \\
\mathbf{u}_{i+1}=\left(\mathbf{H v}_{i}-\alpha_{i} \mathbf{u}_{i}\right) / \beta_{i+1}, \\
\mathbf{v}_{i+1}=\left(\mathbf{H}^{H} \mathbf{u}_{i}-\beta_{i} \mathbf{v}_{i}\right) / \alpha_{i+1}, \\
\text { until } \alpha_{i+1}=0 \text { or } \beta_{i+1}=0 .
\end{gathered}
$$

The vectors $\mathbf{u}_{i}, i=1,2, \ldots$, are orthonormal, and the same is true for the vectors $\mathbf{v}_{i},, i=1,2, \ldots$. They allow to reduce the approximation problem over the $i$ th Krylov subspace to the LS problem

$$
\min _{\mathbf{w}_{i}}\left\|\mathbf{B}_{i} \mathbf{w}_{i}-\beta_{1} \mathbf{e}_{1}\right\|,
$$

where $\mathbf{e}_{1}=\left[\begin{array}{llll}1 & 0 & \ldots & 0\end{array}\right]^{T}$ and $\mathbf{B}_{i}$ is the $(i+1) \times i$ lower bidiagonal matrix with $\alpha_{1}, \ldots, \alpha_{i}$ on the main diagonal, and $\beta_{2}, \ldots, \beta_{i+1}$ on the first subdiagonal. The $i$ th approximate solution of the original problem is given by $\mathbf{x}_{i}=$ $\left[\mathbf{v}_{1} \ldots \mathbf{v}_{i}\right] \mathbf{w}_{i}$.

The second LSQR step solves the least squares problem (5) using the QR factorization of $\mathbf{B}_{i}$. The computational costs of this step are negligible due to the bidiagonal nature of $\mathbf{B}_{i}$. Furthermore, [17] introduced a simple recursion to compute $\mathbf{w}_{i}$ and $\mathbf{x}_{i}$ via a simple vector update from the approximate solution obtained in the previous iteration.

\section{REFERENCES}

[1] J. A. C. Bingham, "Multicarrier modulation for data transmission: An idea whose time has come," IEEE Comm. Mag., vol. 28, pp. 5-14, May 1990.

[2] IEEE P802 LAN/MAN Committee, "The working group for wireless local area networks (WLANs)." http://grouper.ieee.org/groups/802/11/index.html.
[3] IEEE P802 LAN/MAN Committee, "The working group on broadband wireless access standards." http://grouper.ieee.org/groups/802/16/index.html.

[4] ETSI, "Digital video broadcasting (DVB); framing structure, channel coding and modulation for digital terrestrial television.” EN 300 744, V1.4.1, 2001 (http://www.etsi.org).

[5] ETSI, "Digital audio broadcasting (DAB) to mobile, portable and fixed receivers." ETS 300 401, 1995. http://www.etsi.org.

[6] ETSI, "Digital radio mondiale (DRM): System specification." EN 201 980, V2.1.1, 2004 (http://www.etsi.org).

[7] S. Tomasin, A. Gorokhov, H. Yang, and J. P. Linnartz, "Iterative interference cancellation and channel estimation for mobile OFDM," IEEE Trans. Wireless Comm., vol. 4, pp. 238245, Jan. 2005.

[8] P. Robertson and S. Kaiser, "The effects of Doppler spreads in OFDM(A) mobile radio systems," in Proc. IEEE VTC-1999, (Amsterdam, The Netherlands), pp. 329-333, Sept. 1999.

[9] Y. Li and L. Cimini, "Bounds on the interchannel interference of OFDM in time-varying impairments," IEEE Trans. Comm., vol. 49, pp. 401-404, March 2001.

[10] M. Russell and G. L. Stüber, "Interchannel interference analysis of OFDM in a mobile environment," in Proc. IEEE VTC-95, (Chicago, IL), pp. 820-824, July 1995.

[11] X. Cai and G. B. Giannakis, "Bounding performance and suppressing intercarrier interference in wireless mobile OFDM," IEEE Trans. Comm., vol. 51, pp. 2047-2056, Dec. 2003.

[12] Y.-S. Choi, P. J. Voltz, and F. A. Cassara, "On channel estimation and detection for multicarrier signals in fast and selective rayleigh fading channels," IEEE Trans. Comm., vol. 49, pp. 1375-1387, Aug. 2001.

[13] L. Rugini, P. Banelli, and G. Leus, "Simple equalization of time-varying channels for OFDM," IEEE Comm. Letters, vol. 9, pp. 619-621, July 2005.

[14] P. Schniter, "Low-complexity equalization of OFDM in doubly-selective channels," IEEE Trans. Signal Processing, vol. 52, pp. 1002-1011, April 2004.

[15] W. G. Jeon, K. H. Chang, and Y. S. Cho, "An equalization technique for orthogonal frequency-division multiplexing systems in time-variant multipath channels," IEEE Trans. Comm., vol. 47, pp. 27-32, Jan. 1999.

[16] P. C. Hansen, ed., Rank-Deficient and Discrete Ill-Posed Problems: Numerical Aspects of Linear Inversion. Philadelphia (PA): SIAM, 1998.

[17] C. C. Paige and M. a. Saunders, "LSQR: An algorithm for sparse linear equations and sparse least squares," ACM Trans. Math. Software, vol. 8, pp. 43-71, March 1982.

[18] G. H. Golub and C. F. Van Loan, Matrix Computations. Baltimore: Johns Hopkins University Press, 3rd ed., 1996.

[19] P. A. Bello, "Characterization of randomly time-variant linear channels," IEEE Trans. Comm. Syst., vol. 11, pp. 360-393, 1963.

[20] D. Schafhuber, G. Matz, and F. Hlawatsch, "Predictive equalization of time-varying channels for coded OFDM/BFDM systems," in Proc. IEEE GLOBECOM-2000, (San Franscisco, CA), pp. 721-725, Nov./Dec. 2000.

[21] A. M. Sayeed and B. Aazhang, "Joint multipath-Doppler diversity in mobile wireless communications," IEEE Trans. Comm., vol. 47, pp. 123-132, Jan. 1999. 